\title{
Global dynamics of the Mixmaster model
}

\author{
Alan D. Rendall \\ Max-Planck-Institut für Gravitationsphysik \\ Schlaatzweg 1 \\ 14473 Potsdam \\ Germany
}

\begin{abstract}
The asymptotic behaviour of vacuum Bianchi models of class A near the initial singularity is studied, in an effort to confirm the standard picture arising from heuristic and numerical approaches by mathematical proofs. It is shown that for solutions of types other than VIII and IX the singularity is velocity dominated and that the Kretschmann scalar is unbounded there, except in the explicitly known cases where the spacetime can be smoothly extended through a Cauchy horizon. For types VIII and IX it is shown that there are at most two possibilities for the evolution. When the first possibility is realized, and if the spacetime is not one of the explicitly known solutions which can be smoothly extended through a Cauchy horizon, then there are infinitely many oscillations near the singularity and the Kretschmann scalar is unbounded there. The second possibility remains mysterious and it is left open whether it ever occurs. It is also shown that any finite sequence of distinct points generated by iterating the Belinskii-Khalatnikov-Lifschitz mapping can be realized approximately by a solution of the vacuum Einstein equations of Bianchi type IX.
\end{abstract}

\section{Introduction}

Solutions of the vacuum Einstein equations with an $S U(2)$ isometry group acting on spacelike hypersurfaces have been studied extensively over the past twenty-five years or more. This class of spacetimes was christened the 'Mixmaster model' by Misner. A useful point of entry into the literature on the subject is the book of Hobill et. al. [11], where many of the contributions are devoted to the Mixmaster model. Almost all the work which has been done in this area is either heuristic in nature or based on numerical calculations. There are very few rigorous results. Although the numerical and heuristic approaches have led to differences of opinion, it seems that by now a consensus has developed concerning various features of the evolution of these spacetimes. This will be referred to in the following as the 'standard picture'. This paper is an investigation of what aspects of this standard picture can be supported by rigorous theorems.

There are different motivations which explain the amount of effort which has been put into understanding this very special class of spacetimes. One of these is the desire to understand the nature of spacetime singularities and in particular to find out whether 
the geometry near a singularity admits a simple description or whether it is intrinsically complicated. The interest of the Mixmaster model is that it is a case where although the setting is relatively simple (the Einstein equations reduce to ordinary differential equations) the behaviour of the solutions seems to be very complicated. Another motivation for studying the Mixmaster model is provided by the idea of Belinskii, Khalatnikov and Lifschitz [3] that it could provide an approximate description for very general spacetime singularities. This suggestion that very general spacetime singularities can be described approximately by a relatively simple model, if true, is only useful insofar as the model itself is understood.

In this paper the ordinary differential equations which describe the Mixmaster model will be looked at from the point of view of the theory of dynamical systems. This approach has been discussed extensively in the book of Bogoyavlensky [5]. This book contains many interesting ideas, but does not contain any general theorems on the Mixmaster model, the end result being an impressionistic informal description as to what the behaviour of generic solutions should be. Another way of writing the equations for the Mixmaster model as a dynamical system has been presented by Wainwright and Hsu [22]. Their equations cover a wider class of spacetimes which are spatially homogeneous. In particular they include vacuum spacetimes of Bianchi types of class $\mathrm{A}$, i.e. Bianchi types $\mathrm{I}, \mathrm{II}, \mathrm{VI}_{0}, \mathrm{VII}_{0}$, VIII and IX. This means considering spacetimes with three-dimensional isometry groups more general than just $S U(2)$. The type IX spacetimes constitute the Mixmaster model. According to the standard picture general spacetimes of type VIII have a complicated singularity similar to that of general spacetimes of type IX while the singularities in solutions of the other types are much simpler. It turns out to be very helpful to study all class A models in a unified way rather that trying to handle type IX in isolation. In [22] various general properties of this system were determined, and in particular the nature of its critical points was investigated in detail. The work of Wainwright and Hsu is the starting point for the investigations of the present paper.

The results of the paper can be summarized as follows. Any solution of the vacuum Einstein equations with a Bianchi symmetry of class A can be classified, according to its behaviour near the initial singularity, into one of three types. These will be referred to as 'standard convergent', 'standard oscillatory' and 'anomalous'. (For the precise meaning of this terminology, see the last paragraph of Section 4). Solutions of types I, II, $\mathrm{VI}_{0}$ and $\mathrm{VII}_{0}$ belong to the standard convergent type. This is also true of the NUT solutions of types VIII and IX, discussed further below. All other solutions of types VIII and IX belong to either the standard oscillatory or the anomalous type. If it could be shown that the anomalous type never occurs then this would provide a strong confirmation of what was referred to above as the standard picture. However, the results of this paper do not suffice to obtain that conclusion; in fact they do not even suffice to show that the standard oscillatory type ever occurs.

The detailed conclusions which can be made about the spacetimes of the different types just introduced concern on the one hand whether the singularity is a curvature singularity and on the other hand precise statements about the convergent or oscillatory nature of the spacetimes near the initial singularity. Those vacuum Bianchi models of class A which admit an extension through a smooth Cauchy horizon have been determined explicitly in 
[6]. Here it is shown that in any vacuum spacetime of Bianchi class A which does not admit such an extension, and which is not anomalous, the Kretschmann scalar $R_{\alpha \beta \gamma \delta} R^{\alpha \beta \gamma \delta}$ is unbounded in a neighbourhood of the initial singularity. This can be seen as a result on the non-existence of 'intermediate singularities' in this class of spacetimes. In general this result is weakened by the fact that we have no control over how many anomalous spacetimes exist. On the other hand, for types other than VIII and IX it provides optimal information. As for the question of convergent or oscillatory behaviour, it is shown that in the standard convergent case the singularity is velocity dominated. On the other hand, in the standard oscillatory case it is shown that naturally defined geometrical quantities undergo infinitely many oscillations as the singularity is approached.

One aspect of the standard picture is that general solutions of the Mixmaster model are supposed to be approximated in some sense near the singularity by a mapping of the circle to itself, the BKL (Belinskii-Khalatnikov-Lifschitz) mapping. The results on oscillations mentioned up to now say nothing about this. However it can be proved to be true in a weak sense. The statement is that, given any finite sequence generated by the BKL map, there exists a solution of the Einstein vacuum equations of type IX (or indeed of type VIII) which reproduces this sequence with any desired degree of accuracy.

The paper is organized as follows. Section 2 presents some parts of the theory of dynamical systems which will be necessary in the analysis. In Section 3 the system of Wainwright and Hsu is recalled. Various results on the asymptotic behaviour of a solution of the Wainwright-Hsu system as the singularity is approached are proved in Section 4. In Section 5 these results are used to obtain conclusions about curvature singularities and the convergent or oscillatory behaviour of the corresponding spacetime. Moreover, it contains the precise formulations of the results which have been presented informally in the preceding paragraphs.

\section{Background on dynamical systems}

The purpose of this section is to collect together some facts on dynamical systems which are useful in the analysis of the dynamics of Bianchi models. For a more general introduction to relevant aspects of the theory the reader is referred to [21]. Consider the system of ordinary differential equations:

$$
\frac{d x}{d t}=f(x)
$$

where $f: \mathbf{R}^{n} \rightarrow \mathbf{R}^{n}$ is a $C^{\infty}$ mapping. (The choice of the differentiability class $C^{\infty}$ is not essential in what follows; it suffices for the applications in this paper.) The corresponding local flow is the mapping $F$ defined by the condition that $F(t, x)$ is the value at time $t$ of the solution of (2.1) which takes on the value $x$ at time $t=0$, provided the solution exists that long. A critical point of (2.1) is a point $x$ with $f(x)=0$. An important element in the analysis of the global properties of solutions of (2.1) is the study of the local behaviour of solutions near critical points. This can be described using the concept of topological equivalence.

Definition If $x_{0}$ is a critical point of (2.1) and $y_{0}$ is a critical point of the system $d y / d t=$ $g(y)$ with local flow $G(t, y)$, then the two systems are said to be topologically equivalent 
near the points $x_{0}$ and $y_{0}$ respectively if there exists a homeomorphism $\phi$ of an open neighbourhood $U$ of $x_{0}$ onto an open neighbourhood $V$ of $y_{0}$, with $\phi\left(x_{0}\right)=y_{0}$ such that whenever $F(t, x)$ is defined for some $x \in U, G(t, \phi(x))$ is also defined, and is equal to $\phi(F(t, x))$.

In other words, when expressed in appropriate local coordinates the solutions of the two systems look identical. A critical point $x_{0}$ of the system (2.1) is called hyperbolic if the derivative $D f\left(x_{0}\right)$ of $f$ at that point (which defines the linearization of the system about that point) has no eigenvalues which are purely imaginary. A fundamental result on hyperbolic critical points is the Hartman-Grobman theorem ([9], p. 244):

Theorem 2.1 If $x_{0}$ is a hyperbolic critical point of the system (2.1) then the system is topologically equivalent near $x_{0}$ to the linearized system $d \bar{x} / d t=D f\left(x_{0}\right) \bar{x}$ near the origin.

Note that the homeomorphism whose existence is required in the definition of topological equivalence cannot in general be chosen to be a diffeomorphism. However this fact will play no role in what follows. Linear systems which have no purely imaginary eigenvalues can be classified up to topological equivalence ([2], p. 48). Let $n_{+}$and $n_{-}$be the number of eigenvalues with positive and negative real parts respectively. Two linear systems of ODE without purely imaginary eigenvalues are topologically equivalent near the origin if and only if the corresponding values of $n_{+}$and $n_{-}$are equal. (In fact in this case the equivalence is global, i.e. $U$ and $V$ can be taken to be all of $\mathbf{R}^{n}$.) Thus up to topological equivalence, the only linear systems on $\mathbf{R}^{n}$ with no purely imaginary eigenvalues are given by the systems on $\mathbf{R}^{n_{+}} \times \mathbf{R}^{n_{-}}$defined by $d y / d t=y, d z / d t=-z$, with $y \in \mathbf{R}^{n_{+}}$and $z \in \mathbf{R}^{n_{-}}$. A system of ODE of this type is known as a standard saddle and there are only $n+1$ possibilities in $\mathbf{R}^{n}$. If $n_{+}$or $n_{-}$vanishes then there is not a saddle in the usual sense, but rather a source or sink. Nevertheless, we include this case in the definition of a standard saddle. Combining this discussion with the Hartman-Grobman theorem shows that in a neighbourhood of any hyperbolic critical point the system (2.1) is topologically equivalent to a standard saddle.

What can be said in the case of a critical point $x_{0}$ which is not hyperbolic? Let $E_{+}$, $E_{0}$ and $E_{-}$be the spaces spanned by those generalized eigenvectors of $\operatorname{Df}\left(x_{0}\right)$ whose real parts are positive, zero and negative respectively. These are called the unstable, centre and stable subspaces respectively. In general $\mathbf{R}^{n}$ is the direct sum of these three subspaces and the hyperbolic case is that where the centre subspace reduces to zero. Associated to these subspaces are locally invariant manifolds. A submanifold $M$ of an open neighbourhood $U$ of $x_{0}$ is called locally invariant if whenever $x \in M$ and $F(t, x) \in U$ then $F(t, x) \in M$.

Definition A (local) stable, centre or unstable manifold of the critical point $x_{0}$ is a $C^{1}$ submanifold of an open neighbourhood $U$ of $x_{0}$ which is locally invariant, which contains $x_{0}$, and whose tangent space at $x_{0}$ is the stable, centre or unstable subspace respectively.

From the topological classification of hyperbolic fixed points discussed above it is clear that a hyperbolic fixed point has stable and unstable manifolds which are locally unique, i.e. the intersection of any such manifold with a sufficiently small neighbourhood of the 
critical point is unique. A generalization of this to non-hyperbolic critical points is given by the centre manifold theorem [1]:

Theorem 2.2 Let $x_{0}$ be a critical point of (2.1) and suppose that $f$ is $C^{\infty}$. Then there exist stable and unstable manifolds of class $C^{\infty}$ and a centre manifold of class $C^{k}$ for any finite $k$. The stable and unstable manifolds are locally unique.

The centre manifold need not be $C^{\infty}$ in general; the $C^{k}$ centre manifolds may shrink as $k$ increases. The centre manifold need also not be locally unique. (See [23], p. 210, for an example). Despite the non-uniqueness of the centre manifold, it can be used to formulate a generalization of the Hartman-Grobman theorem to non-hyperbolic critical points. This is the reduction theorem of Shoshitaishvili ([18], [19]). For a detailed proof in English see $[13]$.

Theorem 2.3 Let $x_{0}$ be a critical point of (2.1). Then the system is topologically equivalent near $x_{0}$ to the Cartesian product of a standard saddle with the restriction of the flow to any centre manifold.

Suppose for a moment that $f$ is such that all solutions of (2.1) can be extended so as to be defined for all real values of $t$. Then an $\alpha$-limit point of a solution $x(t)$ of the system (2.1) is a point $x_{*}$ such that there exists a sequence $t_{n}$ with $t_{n} \rightarrow-\infty$ and $x\left(t_{n}\right) \rightarrow x_{*}$. The $\alpha$-limit set of the solution is the set of all $\alpha$-limit points. The concepts of $\omega$-limit point and $\omega$-limit set are defined analogously by replacing $-\infty$ by $\infty$. If not all solutions of (2.1) extend to global in time solutions it is possible to rescale $f$ with a positive function so that all solutions of the rescaled system do exist globally in time. This is discussed by Wainwright and $\mathrm{Hsu}[22]$, section 3. The notions of $\alpha$ - and $\omega$-limit points can be applied to the rescaled system. The image of a solution of the original system in $\mathbf{R}^{n}$ is also the image of a solution of the rescaled system. It is natural to define the $\alpha$ - and $\omega$ - limit points of a solution $x(t)$ of the original system to be those of the corresponding solution of the rescaled system. This definition can be rephrased in terms of the original system. A solution $x(t)$ of (2.1) defined on some time interval is said to be maximally extended if it cannot be extended to a solution on any strictly longer interval. If a maximally extended solution $x(t)$ is defined on the interval $\left(t_{-}, t_{+}\right)$, then the $\alpha$-limit and $\omega$-limit points of the solution are limits of sequences of the form $x\left(t_{n}\right)$ where $t_{n} \rightarrow t_{-}$or $t_{n} \rightarrow t_{+}$. This is consistent with the previous definition in the case that the solution is defined for all real values of $t$.

Some standard properties of $\alpha$-limit sets will now be listed (cf. [23], p. 46) Corresponding properties hold for $\omega$-limit sets. The $\alpha$-limit set of any solution is closed. It consists of a union of images of solutions of the ODE. It is clear that it must also contain the $\alpha$-limit sets of these solutions. If the solution stays in a compact set for all $t<t_{0}$ then the $\alpha$-limit set is connected. Monotone functions are a useful tool for locating $\alpha$ - and $\omega$-limit sets, as shown by the following simple lemma.

Lemma 2.1 Let $U$ be an open subset of $\mathbf{R}^{n}$ and let $F$ be a continuous function on $U$ such that $F(f(t))$ is strictly monotone for any solution $f(t)$ of $(2.1)$ as long as $f(t)$ is in in $U$. Then no solution of (2.1) whose image is contained in $U$ has an $\alpha$ - or $\omega$-limit point in $U$. 
Proof Suppose that $p \in U$ is an $\alpha$-limit point of a solution $f(t)$ whose image is contained in $U$. Then $F(f(t))$, being strictly monotone, must have a limit, possibly infinite, as $t \rightarrow t_{-}$. On the other hand, there is a sequence $t_{n}$ with $t_{n} \rightarrow t_{-}$such that $F\left(f\left(t_{n}\right)\right)$ coverges to $F(p)$. Hence $F(f(t))$ converges to $F(p)$. Thus $F$ is constant on the $\alpha$-limit set of $f(t)$. There exists a solution $\bar{f}(t)$ which passes through $p$ and is entirely contained in the $\alpha$-limit set of $f(t)$. It follows from the above that $F$ is constant along $\bar{f}(t)$, contradicting the property of strict monotonicity. The argument for the $\omega$-limit set is similar.

\section{The equations of Wainwright and Hsu}

There are many popular ways of writing the equations for Bianchi models. The analysis which follows uses a form of the equations for models of class A due to Wainwright and Hsu [22]. This is natural since it is in a sense an extension of their approach. The results of the present paper concern vacuum spacetimes, whereas the Wainwright-Hsu system describes a perfect fluid with linear equation of state $p=(\gamma-1) \rho$. However, vacuum models are described by the restriction to the submanifold defined by the vacuum Hamiltonian constraint. The latter is independent of $\gamma$ and so the choice of $\gamma$ is immaterial for our purposes. However, the explicit form of the equations off the constraint hypersurface is more or less complicated according to the value of $\gamma$. In order to take advantage of this, $\gamma=2 / 3$ is chosen in the following. With this choice, the equations are:

$$
\begin{aligned}
& N_{1}^{\prime}=\left(q-4 \Sigma_{+}\right) N_{1} \\
& N_{2}^{\prime}=\left(q+2 \Sigma_{+}+2 \sqrt{3} \Sigma_{-}\right) N_{2} \\
& N_{3}^{\prime}=\left(q+2 \Sigma_{+}-2 \sqrt{3} \Sigma_{-}\right) N_{3} \\
& \Sigma_{+}^{\prime}=-(2-q) \Sigma_{+}-3 S_{+} \\
& \Sigma_{-}^{\prime}=-(2-q) \Sigma_{-}-3 S_{-}
\end{aligned}
$$

where

$$
\begin{aligned}
q & =2\left(\Sigma_{+}^{2}+\Sigma_{-}^{2}\right) \\
S_{+} & =\frac{1}{2}\left[\left(N_{2}-N_{3}\right)^{2}-N_{1}\left(2 N_{1}-N_{2}-N_{3}\right)\right] \\
S_{-} & =\frac{\sqrt{3}}{2}\left(N_{3}-N_{2}\right)\left(N_{1}-N_{2}-N_{3}\right)
\end{aligned}
$$

and a prime denotes a derivative with respect to a certain time coordinate $\tau$. The (vacuum) Hamiltonian constraint is:

$$
\Sigma_{+}^{2}+\Sigma_{-}^{2}+\frac{3}{4}\left[N_{1}^{2}+N_{2}^{2}+N_{3}^{2}-2\left(N_{1} N_{2}+N_{2} N_{3}+N_{3} N_{1}\right)\right]=1
$$

A solution of the vacuum Einstein equations with a Bianchi symmetry of class A is described by a solution of (3.1) with initial data satisfying (3.3). Then of course the whole solution lies in the submanifold defined by (3.3). If $t$ is a Gaussian time coordinate based on one of the orbits of the group action defining the symmetry, and if $\operatorname{tr} k(t)$, denotes the mean curvature of these orbits, then the time coordinate $\tau$ in (3.1) is defined by the relation $\tau(t)=-\frac{1}{3} \int_{t_{0}}^{t} \operatorname{tr} k\left(t^{\prime}\right) d t^{\prime}$. Since the determinant of the induced metric of the orbits satisfies $d / d t(\log \operatorname{det} g)=-2 \operatorname{tr} k, \tau^{3}$ is proportional to the volume form of the orbits. In other 
words $\tau$ represents a length scale related to that volume form. For the precise definition of the variables $\left(N_{1}, N_{2}, N_{3}, \Sigma_{+}, \Sigma_{-}\right)$the reader is referred to [22]. The variables $N_{1}, N_{2}$ and $N_{3}$ describe the curvature of the spatial slices and, at the same, the Bianchi type. In particular they allow all Bianchi types of class A to be included in a single dynamical system. When a fluid is present, $\Sigma_{+}$and $\Sigma_{-}$are related to the shear. In the vacuum case they represent the trace-free part of the second fundamental form. All of these variables are dimensionless, in the sense that if the spacetime metric is multiplied by a constant they do not change. This means that they are candidates for quantities which remain finite when a spacetime singularity is approached. The system (2.3) has a three-fold symmetry, which is not obvious when the equations are written in this form. It is described in detail in [22]. In particular, it cyclically permutes $N_{1}, N_{2}$ and $N_{3}$.

In discussing the global structure of the spacetimes considered in this paper, we restrict to the maximal Cauchy development of data on a spacelike orbit, which is in any case the only part of the spacetime described directly by the variables in (3.1). It is well-known that a vacuum Bianchi model of class A, and not of type IX, is geodesically complete in one time direction which, reversing the time orientation if necessary, we can assume to be the future. In the other time direction there is a finite upper bound to the length of all causal geodesics. In the case of Bianchi type IX, a bound of this kind holds in both directions as a consequence of a result of Lin and Wald[14,15]. In the latter case the variables in (3.1) are not defined at the moment of maximum expansion. For the definition of these variables involves dividing by the mean curvature trk, which vanishes at that moment. Thus a maximally extended solution of (3.1) of Bianchi type IX represents only half of a maximal Cauchy development, where the model is either always expanding or always contracting. Reversing the time orientation if necessary, it can be assumed that it is expanding, so that the singularity lies in the past. With these conventions, if a maximal solution of (3.1) is defined on the interval $\left(\tau_{-}, \tau_{+}\right)$then the behaviour of the corresponding spacetime near the singularity is encoded in the behaviour of the solution as $\tau \rightarrow \tau_{-}$. For Bianchi types other than IX the behaviour as $\tau \rightarrow \tau_{+}$encodes the behaviour of the spacetime in the time direction where it is complete. For Bianchi type IX it encodes the behaviour near the time of maximal expansion.

The Bianchi type to which a solution of (3.1) corresponds depends on the values of $N_{1}-N_{3}$. If all three are zero the Bianchi type is I. If precisely one is non-zero then it is II. If precisely two are non-zero it is either $\mathrm{VI}_{0}$ (signs opposite) or $\mathrm{VII}_{0}$ (signs equal). If all three are non-zero it is either IX (all signs equal) or VIII (one sign different from the other two). The set of points corresponding to any one of the Bianchi types is invariant under the flow of the differential equation. A point of this set will be referred to as a point of the given Bianchi type.

There are various monotone functions which can be defined. The function $N_{1} N_{2} N_{3}$ is strictly monotone along any solution of type IX or VIII. (Cf. the function $\Delta_{1}$ of [22].) For $\left(N_{1} N_{2} N_{3}\right)^{\prime}=3 q\left(N_{1} N_{2} N_{3}\right)$. This gives the result for $q \neq 0$. If $q=0$ then the Hamiltonian constraint shows that either $\Sigma_{+}^{\prime}$ or $\Sigma_{-}^{\prime}$ is non-zero and this proves the desired result. The functions

$$
Z_{\epsilon}=\left[\frac{4}{3} \Sigma_{-}^{2}+\left(N_{2}+\epsilon N_{3}\right)^{2}\right] /\left(-\epsilon N_{2} N_{3}\right)
$$

are non-negative functions on the sets of points of type $\mathrm{VI}_{0}$ and $\mathrm{VII}_{0}$ with $N_{1}=0$ for 
$\epsilon=1$ and $\epsilon=-1$ respectively. They are strictly decreasing except when $\Sigma_{-}=0$. These statements are proved in [22]. The function $\left(1+\Sigma_{+}\right)^{2}$ is a non-increasing function along solutions on the union of points of types I, II, $\mathrm{VI}_{0}$ and $\mathrm{VII}_{0}$ with $N_{1}=0$. For

$$
d / d \tau\left[\left(1+\Sigma_{+}\right)^{2}\right]=-4\left(1+\Sigma_{+}\right)^{2}\left(1-\Sigma_{+}^{2}-\Sigma_{-}^{2}\right)
$$

and the vacuum Hamiltonian constraint implies that $\Sigma_{+}^{2}+\Sigma_{-}^{2} \leq 1$ for the given Bianchi types. This is analogous to a monotone function for solutions of class B given by Hewitt and Wainwright [10].

In order to study the question of which of the spacetimes described by the above equations have curvature singularities, it is useful to express curvature invariants of the spacetimes in terms of the Wainwright-Hsu variables. Consider the example of the Kretschmann scalar $R_{\alpha \beta \gamma \delta} R^{\alpha \beta \gamma \delta}$. Define a dimensionless version of this quantity by

$$
\kappa=R_{\alpha \beta \gamma \delta} R^{\alpha \beta \gamma \delta} /(\operatorname{tr} k)^{4}
$$

Then $\kappa$ can be expressed as a polynomial in the variables $N_{1}, N_{2}, N_{3}, \Sigma_{+}$and $\Sigma_{-}$. This can conveniently be done using the formulae for curvature given by Ellis and MacCallum [8]. Here only the expression for solutions of type I will be written out. In that case:

$$
\kappa=\left(-\frac{2}{9}+\frac{2}{9} \Sigma_{+}+\frac{4}{9} \Sigma_{+}^{2}\right)^{2}+2\left(\frac{1}{9} \Sigma_{+}^{2}+\frac{1}{3} \Sigma_{-}^{2}-\frac{1}{9} \Sigma_{+}-\frac{2}{9}\right)^{2}+\frac{2}{27} \Sigma_{-}^{2}\left(2 \Sigma_{+}-1\right)^{2}
$$

The fact that it is a positive definite expression is explained by the fact that for a vacuum spacetime of Bianchi type I the magnetic part of the Weyl tensor vanishes.

\section{Possible limit sets}

It will now be shown among other things that the $\alpha$-limit set of any solution of (3.1)(3.3) of type I, II, $\mathrm{VI}_{0}$ or $\mathrm{VII}_{0}$ is a single point of type I or type $\mathrm{VII}_{0}$. Consider first a solution of type I. It corresponds to a critical point of (3.1) and so is its own $\alpha$-limit set, as well as its own $\omega$-limit set. These points form a circle, sometimes known as the Kasner ring. Next consider a solution of type II. Using the threefold symmetry of the equations, it may be assumed without loss of generality that it satisfies $N_{2}=N_{3}=0$. Then an elementary calculation shows that either $\Sigma_{-}=0$ or the ratio $\left(\Sigma_{+}-2\right) / \Sigma_{-}$is time independent. Taking account of the Hamiltonian constraint, this reduces the motion to motion along a curve. The direction of motion along this curve is controlled by the monotone function $\left(1+\Sigma_{+}\right)^{2}$. It follows that the $\alpha$-limit set consists of one point on the Kasner ring and the $\omega$-limit set of another. There are three points on the Kasner ring which play a special role. They are denoted by $T_{1}, T_{2}$ and $T_{3}$ in [22] and have coordinates $(-1,0),(1 / 2, \pm \sqrt{3} / 2)$. They divide the Kasner ring into three equal parts. The $\alpha$-limit set of a solution of type II with $N_{1} \neq 0$ lies on the longer of the two open arcs with endpoints $T_{2}$ and $T_{3}$, while the $\omega$-limit set lies on the shorter of these $\operatorname{arcs}$. The points $T_{1}, T_{2}$ and $T_{3}$ are permuted cyclically by the threefold symmetry, which shows what happens for type II solutions with $N_{2} \neq 0$ or $N_{3} \neq 0$. Associating the $\alpha$-limit set of a type II solution with the $\omega$-limit set of that solution defines a mapping from the Kasner ring with the three exceptional points removed to the Kasner ring. This is a realization of the famous BKL (Belinskii-Khalatnikov-Lifshitz) mapping. 
Consider next solutions of type $\mathrm{VI}_{0}$ or $\mathrm{VII}_{0}$. Using the threefold symmetry, it can be assumed without loss of generality that $N_{1}=0$. Equation (3.4) and Lemma 2.1 imply that for any $\alpha$-limit point $\Sigma_{+}^{2}+\Sigma_{-}^{2}=1$ or $\Sigma_{+}=-1$. For these solutions the Hamiltonian constraint reduces to:

$$
\Sigma_{+}^{2}+\Sigma_{-}^{2}+\frac{3}{4}\left(N_{2}-N_{3}\right)^{2}=1
$$

and so if $\Sigma_{+}=-1$ then $\Sigma_{+}^{2}+\Sigma_{-}^{2}=1$ anyway. Moreover $N_{2}=N_{3}$. This means in particular that there are no $\alpha$-limit points of type $\mathrm{VI}_{0}$. The $\alpha$-limit set must contain a solution of the equations, which satisfies these conditions everywhere. The second and third equations of (3.1) then give either $N_{2}=N_{3}=0$ or $\Sigma_{-}=0$, whence $\Sigma_{+}= \pm 1$. Where $\Sigma_{+}=-1, \Sigma_{+}+1=0$ and since this function is non-negative and non-increasing along solutions, it follows that any solution which has a point of this type as an $\alpha$-limit point must be a time independent solution. Apart from these time-independent solutions, the remaining possibilities are that either the $\alpha$-limit set contains a point with $\Sigma_{+}=1$, $\Sigma_{-}=0$ and $N_{2}=N_{3}$, or that the entire $\alpha$-limit set is contained in the Kasner ring. The points in the first of these two cases constitute the image of a whole solution and so if one of them belongs to the $\alpha$-limit set of a solution, they all do. In particular, this would mean that the solution would not remain in a compact set. It will now be seen that this is impossible. In the Bianchi $\mathrm{VI}_{0}$ case this is simple, since the Hamiltonian constraint shows that the set of Bianchi $\mathrm{VI}_{0}$ points is compact. The Bianchi $\mathrm{VII}_{0}$ case requires a little more work. Consider the behaviour of the non-increasing function $Z_{-1}$ along any solution of type $\mathrm{VII}_{0}$. Either it is identically zero or or it is bounded below by a positive constant for all $\tau \leq \tau_{0}$ and some fixed $\tau_{0}$. In the first case $N_{2}=N_{3}, \Sigma_{-}=0$ and $\Sigma_{+}= \pm 1$. Then $N_{2}^{\prime}=2\left(1+\Sigma_{+}\right) N_{2}$ and the solution remains in a compact set as $\tau \rightarrow-\tau_{-}$. In the second case, for any fixed $\tau_{0}$,

$$
N_{2} N_{3} \leq C\left[\frac{4}{3} \Sigma_{-}^{2}+\left(N_{2}-N_{3}\right)^{2}\right]
$$

holds for all $\tau \leq \tau_{0}$. Combining this with the Hamiltonian constraint (4.1) proves that the solution remains in a compact set as $\tau \rightarrow-\tau_{-}$. The fact that solutions of these types remain in a compact set for all sufficiently negative times also proves that their $\alpha$-limit sets are non-empty.

The points $T_{1}, T_{2}$ and $T_{3}$ of the Kasner ring correspond to spacetimes obtained by making identifications in a subset of Minkowski space. In particular they are flat. In fact they are the only flat Kasner solutions, since from (3.6) the Kretschmann scalar is nonzero at all other points of the Kasner ring. The points of type of type $\mathrm{VII}_{0}$ which satisfy $\Sigma_{+}=-1$ and $\Sigma_{-}=0$ represent the same spacetime as the point $T_{1}$. The reason for this is that the Wainwright-Hsu variables depend not only on the spacetime but on a choice of frame. The flat Kasner solutions admit a frame of type I and a one-parameter family of frames of type $\mathrm{VII}_{0}$, which can all be used. Thus one spacetime is represented by different points in $\mathbf{R}^{5}$. These type $\mathrm{VII}_{0}$ solutions and those obtained from them by applying the threefold symmetry will be referred to as the flat type $\mathrm{VII}_{0}$ solutions. This terminology will be justified later, when it is shown that they are the only flat solutions of type $\mathrm{VII}_{0}$.

It has now been shown that, except for the flat solutions of type $\mathrm{VII}_{0}$, the $\alpha$-limit set of any solution of type $\mathrm{I}, \mathrm{II}, \mathrm{VI}_{0}$ or $\mathrm{VII}_{0}$ is contained in the Kasner ring. It will now be shown that it consists of a single point of the Kasner ring. To see this, consider the behaviour of the function $\left(1+\Sigma_{+}\right)^{2}$ along the given solution. It cannot take two different 
values on the $\alpha$-limit set, since then it could not be monotonic along the solution. Thus the $\alpha$-limit set of any given solution consists of points on the Kasner ring where $\Sigma_{+}$takes on the same value. But for any given value of $\Sigma_{+}$there is only one point, in which case the desired result follows, or there are two. In the latter case it follows from the connectedness of the $\alpha$-limit set that only one of the two can be contained in it. The following theorem has now been proved:

Theorem 4.1 The $\alpha$-limit set of a solution of (3.1)-(3.3) of type I, II, $\mathrm{VI}_{0}$ or $\mathrm{VII}_{0}$ is a single point of type $\mathrm{I}$ or a flat point of type $\mathrm{VII}_{0}$, the latter only being possible if the solution is time independent.

Note that all the arguments used to prove this were elementary, and that the reduction theorem (Theorem 2.3) was not used. In studying the $\alpha$-limit sets of solutions of types VIII and IX the heavier machinery is required. Note first that, due to the strictly monotone function for these Bianchi types presented in Section 3, the $\alpha$-limit set of any solution of type VIII or IX is contained in the set $N_{1} N_{2} N_{3}=0$, which consists of points of the other, simpler, Bianchi types. It follows that the $\alpha$-limit set, if non-empty, contains the image of a solution of one of these types. It then also contains the $\alpha$-limit set of that solution. Thus it follows from Theorem 4.1 that:

Theorem 4.2 The $\alpha$-limit set of a solution of (3.1)-(3.3) of type VIII or IX is either empty or contains a point of type $\mathrm{I}$ or contains a flat point of type $\mathrm{VII}_{0}$.

In the rest of this section it will be shown that, except for a small set of well-understood solutions, if the $\alpha$-limit set of a solution of type VIII or IX is non-empty, then it either contains a non-empty open subset of flat points of type $\mathrm{VII}_{0}$ or a non-flat point of the Kasner ring and that in the latter case it contains more than one non-flat point of the Kasner ring.

Theorem 4.3 The $\alpha$-limit set of a solution of (3.1)-(3.3) of type VIII or IX has at least one of the following properties:

(i) it is empty

(ii) it contains at least two distinct points of type I, at least one of which is non-flat

(iii) it consists of flat points of type $\mathrm{I}_{\text {or }} \mathrm{VII}_{0}$ and contains a non-empty open subset of the set of points of type $\mathrm{VII}_{0}$.

(iv) it is a flat point of type $\mathrm{I}$ or $\mathrm{VII}_{0}$ and the solution is contained in the unstable manifold of that point

Proof The essential point is to apply the reduction theorem (Theorem 2.3) to the critical points of the system. In this context it is important to note that the Kasner ring is a centre manifold for each of the non-flat points contained in it, while the manifolds $N_{1}=0$, $N_{2}=0$ and $N_{3}=0$ are centre manifolds for the flat solutions of type I and $\mathrm{VII}_{0}$ which they contain. This follows from the computation of the eigenvalues and eigenspaces of the critical points carried out by Wainwright and Hsu [22] and the obvious fact that the manifolds in question are invariant manifolds.

Consider now a solution of type VIII or IX with non-empty $\alpha$-limit set. First it will be 
shown that if its $\alpha$-limit set contains one non-flat point $p$ of type I, it contains at least two such points. The Kasner ring, which is a centre manifold for $p$, consists of critical points. Hence, by the reduction theorem, a neighbourhood of $p$ is foliated by invariant manifolds of codimension one, each of which meets the Kasner ring in a single point. In each of these submanifolds the flow is topologically equivalent to a standard saddle. It follows that if the solution converges to $p$ as $\tau \rightarrow \tau_{-}$, it must lie on the unstable manifold of $p$ and that otherwise the $\alpha$-limit set must contain the stable manifold. Suppose without loss of generality that $p$ belongs to the shorter of the two arcs of the Kasner ring joining $T_{2}$ to $T_{3}$. Then the analysis of the linearization in [22] shows that the subspaces $N_{2}=N_{3}=0$ and $N_{1}=0$ are the stable and unstable manifolds respectively. Since the unstable manifold contains no points of types VIII or IX, it follows that the $\alpha$-limit set of the solution under consideration contains the stable manifold, which is the image of a solution of type II. Since the $\alpha$-limit set is closed, it follows that it must also contain the image of $p$ under the BKL map. Thus it contains at least two distinct points.

Next the case will be considered where no $\alpha$-limit point of the solution is a non-flat point of type I. Because of the monotone function $N_{1} N_{2} N_{3}$, all $\alpha$-limit points must satisfy $N_{1}=0, N_{2}=0$ or $N_{3}=0$. A $\alpha$-limit point of type II or type $\mathrm{VI}_{0}$ or a non-flat point of type $\mathrm{I}$ or $\mathrm{VII}_{0}$ is not possible, as follows from Theorem 4.1. Hence the entire $\alpha$-limit set consists of flat points of type I or $\mathrm{VII}_{0}$. Note also that no solution on the centre manifold can approach one of these points as $\tau \rightarrow \tau_{-}$, unless it is the solution which stays at that point all the time. Hence, by the reduction theorem, the solution under consideration can only approach a point of that type if it lies on the stable manifold of that point. Using the calculations of [22] once more, the stable manifold can be identified. After applying the threefold symmetry if necessary, it is given by the equations $N_{2}=N_{3}, \Sigma_{-}=0$. The Bianchi type IX solutions satifying these conditions are the Taub-NUT solutions. Similarly, solutions of type VIII (or indeed of type II) satisfying these conditions are the NUT solutions of those types described by Siklos[20]. It remains to consider the case of a solution whose $\alpha$-limit points are flat points of type $\mathrm{I}$ or $\mathrm{VII}_{0}$, but which does not converge to such a point. By the reduction theorem, a solution of this type which has $p$ as an $\alpha$-limit point must repeatedly leave the neighbourhood of $p$ whose existence is asserted by the theorem. In particular, it crosses a sphere of any sufficiently small radius about $p$ infinitely many times. Thus some $\alpha$-limit point lies on any such sphere. It follows that the $\alpha$-limit set contains an open subset of the set of flat points of type $\mathrm{VII}_{0}$.

The notions of 'standard convergent', 'standard oscillatory' and 'anomalous' mentioned in the introduction will now be defined in terms of the four cases of the theorem. A solution of type VIII or IX is standard convergent if it satisfies property (iv) of Theorem 4.3. A solution of any other Bianchi type of class $\mathrm{A}$ is always standard convergent. (This terminology is justified by Theorem 4.1.) A solution is standard oscillatory if it satisfies property (ii) of Theorem 4.3. A solution which is neither standard convergent nor standard oscillatory is called anomalous.

\section{Conclusions}

The results of the previous section on the $\alpha$-limit sets of solutions of (3.1)-(3.3) will 
now be interpreted in terms of properties of the corresponding spacetimes. The first issue to be considered is that of curvature singularities. Here the theorems of the last section give complete information for all types other than VIII and IX. For these two types the information obtained is less satisfactory.

Theorem 5.1 Let $(M, g)$ be a vacuum spacetime with a Bianchi symmetry of class A. Suppose that the time orientation has been chosen such that the maximal Cauchy development of data on a homogeneous hypersurface is past incomplete, so that there is a past singularity. Then at least one of the following holds:

(i) the Kretschmann scalar is unbounded in a neighbourhood of the initial singularity

(ii) the maximal Cauchy development can be extended through a smooth Cauchy horizon, and then the spacetime is flat or a NUT spacetime

(iii) the corresponding solution has property (i) or property (iii) of Theorem 4.3

Proof Assume that property (iii) of the conclusions of this theorem does not hold. Then if the solution of (3.1)-(3.3) corresponding to the given spacetime is of type VIII or IX, it has property (ii) or (iv) of the conclusions of Theorem 4.3. The solutions which have property (iv) of Theorem 4.3 have already been identified; they are the NUT spacetimes. It is known that they admit an extension through a smooth Cauchy horizon (cf. [6]). Thus it remains to prove the theorem in the case that a solution of type VIII or IX has property (ii) of Theorem 4.3 and in the case that the Bianchi type is neither VIII nor IX. From Theorems 4.1 and 4.3 it follows that either the spacetime corresponds to a flat point of type $\mathrm{I}$ or $\mathrm{VII}_{0}$ or that there is a non-flat point of type $\mathrm{I}$ in the $\alpha$-limit set. In the first of these cases, it is known that the spacetime can be extended through a smooth Cauchy horizon [6]. In the second case the values of the scalar $\kappa$ introduced in Section 2 have a cluster point $\kappa_{0} \neq 0$ as $\tau \rightarrow \tau_{-}$, where $\kappa_{0}$ is the value of $\kappa$ at a non-flat $\alpha$-limit point of the solution on the Kasner ring. Since the Kretschmann scalar is equal to $\kappa(\operatorname{tr} k)^{4}$, it follows that $\lim \sup R^{\alpha \beta \gamma \delta} R_{\alpha \beta \gamma \delta}=\infty$. This completes the proof.

According the standard picture of the Mixmaster solutions, case (iii) of Theorem 5.1 should not occur. If that were the case, a clean result would be obtained on the non-existence of 'intermediate singularities'. This would mean that every solution possessed either a smooth Cauchy horizon or an unbounded curvature invariant near the singularity. Note that this conclusion has been proved for solutions of type other than VIII and IX, as can be seen from an examination of the above proof. In fact, slightly more has been proved, since it has not only been shown that the Kretschmann scalar is unbounded near the singularity, but also that it tends to infinity there at the same rate as rate $(\operatorname{tr} k)^{4}$. Computing curvature invariants other than the Kretschmann scalar would not lead to an improved result for types VIII and IX using the above techniques, since all curvature invariants vanish at the flat points.

The other important point of interpretation concerns the question of convergent or oscillatory behaviour near the singularity, and velocity dominance. The notion of a velocity dominated singularity was introduced by Eardley, Liang and Sachs [7] and a related notion was used by Isenberg and Moncrief [12] (see also [16], [17] for other applications). The general idea is that near the singularity the spacetime should be approximated in some appropriate sense by a solution of Bianchi type I. In the present context it might be 
tempting to say that a solution of (3.1)-(3.3) was velocity dominated if its $\alpha$-limit set consisted of a point on the Kasner ring. However, this is too simple, since the flat points of type $\mathrm{VII}_{0}$ represent the same spacetimes as the flat points of Bianchi type I. Thus the following definition will be used:

Definition A singularity in a spacetime with a given 3+1-decomposition is called weakly velocity dominated if the generalized Kasner exponents $p_{i}(t, x)$ converge to a limit for each fixed $x$ as the singularity is approached and if their limits satisfy $\Sigma_{i} p_{i}^{2}=1$.

This definition requires some further explanation. Suppose that the hypersurfaces of constant $t$ have nowhere vanishing mean curvature near the singularity. (If this is not the case, the definition is deemed to be violated) Let $\lambda_{i}$ be the eigenvalues of the second fundamental form. Then the mean curvature is $\operatorname{tr} k=\Sigma_{i} \lambda_{i}$ and the generalized Kasner exponents are defined to be $p_{i}=\lambda_{i} /(\operatorname{tr} k)$. In a Kasner solution they are constants and satisfy the equation required in the above definition. For the Bianchi spacetimes considered here, the definition will be applied with a 3+1-decomposition defined by Gaussian coordinates based on a homogeneous hypersurface. Then the generalized Kasner exponents are linear combinations of $\Sigma_{-}$and $\Sigma_{+}$and the equation of the definition becomes $\Sigma_{+}^{2}+\Sigma_{-}^{2}=1$. It is easy to think up alternative definitions. For instance, the condition $\Sigma_{i} p_{i}^{2}=1$ could be replaced by the condition $\rho_{i} /(\operatorname{tr} k)^{2} \rightarrow 0$, where $\rho_{i}$ are the eigenvalues of the spatial Ricci tensor. The resulting definition is a priori stronger than that given above but in all the cases where the above definition is shown to be satisfied in the following the stronger definition is also satsisfied.

Returning to the question of oscillatory behaviour, a function $F$ on an interval $\left(\tau_{-}, \tau_{+}\right)$ will be said to have infinitely many oscillations as $\tau \rightarrow \tau_{-}$if there exist two numbers $a, b$ with $a<b$ and a sequence $\tau_{n}$ with $\tau_{n} \rightarrow \tau_{-}$as $n \rightarrow \infty$ such that for any positive integer $k, F\left(\tau_{2 k-1}\right) \leq a$ and $F\left(\tau_{2 k}\right) \geq b$.

Theorem 5.2 Let $(M, g)$ be a vacuum spacetime with a Bianchi symmetry of class A. Suppose that the time orientation has been chosen such that the maximal Cauchy development of data on a homogeneous hypersurface is past incomplete, so that there is a past singularity. Then either:

(i) the singularity is weakly velocity dominated

(ii) the spacetime is of type VIII or IX and $\Sigma_{+}$or $\Sigma_{-}$has infinitely many oscillations as the singularity is approached.

(iii) the spacetime is of type VIII or IX and the corresponding solution of (3.1)-(3.3) fails to be contained in a compact set as $\tau \rightarrow \tau_{-}$.

Proof If the spacetime is not of type VIII or IX then the result follows immediately from Theorem 4.1. Consider a spacetime of type VIII or IX such the corresponding solution of (3.1)-(3.3) is contained in a compact set as $\tau \rightarrow \tau_{-}$. Then it must have one of the properties (ii), (iii) or (iv) of Theorem 4.3. If it has property (iv) of that theorem the singularity is weakly velocity dominated. If it has property (ii) it repeatedly comes close to two different points of the Kasner ring as $\tau \rightarrow \tau_{-}$and so $\Sigma_{+}$or $\Sigma_{-}$has infinitely many oscillations as the singularity is approached. If it does not belong to any of these cases 
of Theorem 4.2 then its $\alpha$-limit set must be a compact subset of the set of flat points of type $\mathrm{I}$ and $\mathrm{VII}_{0}$. In that case $\Sigma_{+} \rightarrow-1$ and $\Sigma_{-} \rightarrow 0$ and the solution is weakly velocity dominated.

This result implies in particular that solutions which are neither of type VIII or IX are (weakly) velocity dominated. The standard picture indicates that the only solutions of types VIII and IX which are velocity dominated are the NUT solutions and that case (iii) of Theorem 5.1 would be superfluous. However, it has not been proved that this is the case.

Although the BKL mapping plays an implicit role in these results, they do not provide any direct confirmation of the idea, which is part of the standard picture, that the BKL mapping represents an approximation to solutions of the Mixmaster model in some sense. The next result is a statement which goes in that direction. Recall that the BKL mapping is a mapping from the Kasner ring with the three exceptional points $T_{1}, T_{2}$ and $T_{3}$ removed to the Kasner ring. Starting with a point of the Kasner ring and applying the BKL mapping repeatedly produces a sequence of points. If one of these points is one of $T_{1}, T_{2}$ or $T_{3}$ then the map can no longer be applied and only a finite sequence can be defined. If, on the other hand, this never happens, an infinite sequence is obtained. This may or may not be periodic. By a finite non-repeating sequence of BKL iterates we mean a finite initial piece of the iteration, which never hits the same point twice, whether or not the sequence as a whole is finite or infinite for that starting point. In the case that the whole sequence is periodic, we need to cut it off after some number of iterations smaller than the period. To measure the quality of the approximation the Euclidean metric in the space $\mathbf{R}^{5}$ where the Wainwright-Hsu system is defined will be used. The distance between points $x$ and $y$ in this metric will be denoted by $d(x, y)$.

Theorem 5.3 Let $\left\{x_{1}, \ldots, x_{n}\right\}$ be a finite sequence of BKL iterates and $\epsilon>0$. Then there exists a $\delta>0$ and a solution of the vacuum Einstein equations of Bianchi type IX which, when written in the Wainwright-Hsu variables as $f(\tau)$, has the following properties:

1. $\delta<\epsilon$ and the balls of radius $\delta$ about each of the points $x_{i}$ have disjoint closures.

2. There exists a finite sequence of times $\tau_{1}>\ldots>\tau_{n}$ such that $f\left(\tau_{i}\right)$ is contained in the open ball of radius $\delta$ about $x_{i}$ while for $\tau_{i} \leq \tau \leq \tau_{i+1}$ the solution does not come closer than $\delta$ to any $x_{j}$ other than $x_{i}$ or $x_{i+1}$.

Proof As explained in Section 3 each iteration of a point via the BKL map can be represented by a solution of Bianchi type II. For $i=1,2, \ldots, n-1$, let $s_{i}$ be the image of the Bianchi II solution which produces $x_{i+1}$ from $x_{i}$. Choose some $\eta>0$ smaller than $\epsilon$ such that the closures of the balls $B_{i}$ of radius $\eta$ about the points $x_{i}$ are disjoint and such that on each of these balls the flow has the local product structure of the reduction theorem. Now choose $\delta_{0}<\eta$ such that any point which is a distance less than $\delta_{0}$ from each of two distinct $s_{i}$ must be inside one of the balls $B_{i}$. That such a $\delta_{0}$ exists follows from the fact that the intersections of each $s_{i}$ with the complement of the union of the $B_{j}$ are disjoint compact sets. Let $S$ be the set of all points which are a distance less than $\delta_{0}$ from some $s_{i}$. It is an open set which contains all $x_{i}$. For $1 \leq i \leq n-1$ let $y_{i}$ be a point of $s_{i}$ which is a distance less than $\delta_{0}$ from $x_{i}$. For $2 \leq i \leq n$ let $z_{i}$ be a point of $s_{i-1}$ which a distance less 
than $\delta_{0}$ from $x_{i}$. Let $\delta_{1}$ be such that the ball of radius $\delta_{1}$ about $y_{1}$ is contained in the ball of radius $\delta_{0}$ about $x_{1}$. There exists a time $\Delta \tau_{1}$ such that the solution with $f(\tau)=z_{2}$ satisfies $f\left(\tau+\Delta \tau_{1}\right)=y_{1}$. Choose $\epsilon_{2}>0$ such that if $d\left(x, z_{2}\right)<\epsilon_{2}$ then $d\left(F\left(\Delta \tau_{1}, x\right), y_{1}\right)<\delta_{1}$. Here $F$ denotes the local flow of the differential equation. By reducing the size of $\epsilon_{2}$ if necessary, it can be ensured that if $d\left(x, z_{2}\right)<\epsilon_{2}$, then $F(\tau, x) \in S \backslash B_{i}^{\prime}$ for all $\tau \leq \Delta \tau_{1}$, where $B_{i}^{\prime}$ is the complement of $\bar{B}_{i} \cup \bar{B}_{i+1}$. Now choose $\delta_{2}$ such any $x$ with the correct signs of $N_{1}, N_{2}$ and $N_{3}$ which lies in the open ball of radius $\delta_{2}$ about $y_{2}$ passes through the open ball of radius $\epsilon_{2}$ about $z_{2}$ and stays in $S$ as long as it stays in the ball of radius $\delta_{0}$ about $x_{2}$. The existence of a number $\delta_{2}$ with this property follows from the local saddle point structure of the flow near the point $x_{2}$. In a similar way we can recursively define $\Delta \tau_{i}$ for $i=2, \ldots, n-1, \epsilon_{i}$ for $i=3, \ldots, n$ and $\delta_{i}$ for $i=3, \ldots, n-1$. Now let $\delta=\delta_{n-1}$ and let $x_{*}$ be any point of type IX such that $d\left(x_{*}, z_{n}\right)<\delta$. We claim that the solution with initial data $x_{*}$ for $\tau=0$ has the desired properties. Note first that by construction it enters each of the balls $B_{i}$ and is contained in $S$. Let $\tau_{n}=0$. Let $\sigma_{n-1}=\tau_{n}+\Delta \tau_{n-1}$. Let $\tau_{n-1}>\sigma_{n-1}$ be a time such that $d\left(f\left(\tau_{n-1}\right), z_{n-1}\right)<\epsilon_{n-1}$ and $f(\tau)$ is in $B_{i-1}$ for all $\tau$ in the interval $\left[\sigma_{i-1}, \tau_{i-1}\right]$. Continuing in this way, the other $\tau_{i}$ can be defined recursively.

The intuitive meaning of this theorem is that the solution visits small neighbourhoods of the points generated by th BKL iteration and, moreover, does so in the order determined by the iteration. The analogous theorem holds with type VIII replaced by type IX, the proof being almost identical. This result shows that arbitrarily long but finite segments of a BKL iteration can be realized by a solution of the Einstein equations. However, it says nothing about the full iteration, if it happens to be infinite. This is natural, since for a given measure of error $\epsilon$ and a given starting point specified with precision $\epsilon$ it cannot be expected that the qualitative behaviour of the sequence of iterates is determined.

What do Theorems 5.1-5.3 tell us about Mixmaster dynamics? They tell us that a finite sequence of BKL iterates corresponds to a solution of the exact equations. They do not tell us the ultimate fate of the solution at early times because the possibility is left open that eventually all solutions show the behaviour described above as anomalous. Numerical results make this possibility implausible. Recent calculations[4] have been able to observe $150 \mathrm{BKL}$ iterates in numerical solutions of the Mixmaster model. Moreover, there is no indication that things change at that point and anomalous behaviour seems never to have been observed numerically. Thus it seems reasonable to suppose that at least a large open set of initial data for the Mixmaster model gives rise to standard oscillatory behaviour. On the other hand, it it difficult to see how the mathematical techniques used in this paper could lead to a proof of this fact. The key question is, what other techniques might do so.

Acknowledgements I am grateful to J. Isenberg and J. Wainwright for helpful discussions and correspondence.

\section{References}

[1] Abraham R and Robbin J 1967 Transversal mappings and flows (New York: Benjamin) 
[2] Arnold V I and Ilyashenko Yu S 1988 Dynamical Systems I ed D V Anosov and V I Arnold (Berlin: Springer)

[3] Belinskii V A, Khalatnikov I M and Lifshitz E M 1982 A general solution of the Einstein equations with a time singularity. Adv. Phys. 31 639-667

[4] Berger B K, Garfinkle D and Strasser E 1996 New algorithm for Mixmaster dynamics. Preprint gr-qc/9609072

[5] Bogoyavlensky O I 1985 Qualitative theory of dynamical systems in astrophysics and gas dynamics (Berlin: Springer)

[6] Chruściel P T and Rendall A D 1995 Strong cosmic censorship in vacuum spacetimes with compact locally homogeneous hypersurfaces. Ann. Phys. 242 349-385

[7] Eardley D, Liang E. and Sachs R 1972 Velocity-dominated singularities in irrotational dust cosmologies J. Math. Phys. 13 99-106

[8] Ellis G and MacCallum M 1969 A class of homogeneous cosmological models Commun. Math. Phys. 12 108-141

[9] Hartman P 1982 Ordinary Differential Equations (Boston: Birkhäuser)

[10] Hewitt C and Wainwright J 1993 A dynamical systems approach to Bianchi cosmologies: orthogonal models of class B Class. Quantum Grav. 10 99-124

[11] Hobill D, Burd A and Coley A 1994 Deterministic Chaos in General Relativity (New York: Plenum)

[12] Isenberg J and Moncrief V 1990 Asymptotic behaviour of the gravitational field and the nature of singularities in Gowdy spacetimes. Ann. Phys. 199 84-122

[13] Kirchgraber U and Palmer K J 1990 Geometry in the neighborhood of invariant manifolds of maps and flows and linearization (Harlow: Longman)

[14] Lin X-F and Wald R 1989 Proof of the closed universe recollapse conjecture for diagonal Bianchi type IX cosmologies Phys. Rev. D 40 3280-3286

[15] Lin X-F and Wald R 1990 Proof of the closed universe recollapse conjecture for general Bianchi type IX cosmologies Phys. Rev. D 41 2444-2448

[16] Rein G 1996 Cosmological solutions of the Vlasov-Einstein system with spherical, plane and hyperbolic symmetry Math. Proc. Camb. Phil. Soc. 119 739-762

[17] Rendall A D 1995 On the nature of singularities in plane symmetric scalar field cosmologies Gen. Rel. Grav. 27 213-221

[18] Shoshitaishvili A N 1972 Bifurcations of topological type at singular points of parametrized vector fields Funct. Analysis Applications 6 169-170

[19] Shoshitaishvili A N 1975 Bifurcations of topological type at singular points of parametrized vector fields Tr. Semin. I. G. Petrovskii 1 279-309 (Russian)

[20] Siklos S T C 1976 Two completely singularity-free NUT spacetimes. Phys. Lett. A 59 173-174

[21] Wainwright J 1994 Deterministic Chaos in General Relativity ed D Hobill, A Burd and A Coley (New York: Plenum)

[22] Wainwright J and Hsu L 1989 A dynamical systems approach to Bianchi cosmologies: orthogonal models of class A. Class. Quantum Grav. 6 1409-1431

[23] Wiggins S 1990 Introduction to applied nonlinear dynamical systems and chaos. (Berlin: Springer) 